\section{Cognitive Affective Syndrome}

\section{Robert Rider}

Department of Psychology, Drexel University, Philadelphia, PA, USA

\section{Synonyms}

Cerebellar cognitive affective syndrome

\section{Definition}

First described by Schmahmann and Sherman (1998), cerebellar cognitive affective syndrome (CAS) refers to a cluster of impairments involving higher-order cognitive processes and affective functioning. Symptoms tend to cluster in executive dysfunction, including problems with planning, set shifting, verbal fluency, abstract reasoning, perseveration, attentional dysregulation, hyperactivity, impulsivity and disinhibition, and deficits in working memory. However, symptoms may also include visuospatial disorders, expressive language disorders, affective abnormalities, difficulties with visuospatial organization, visual memory, logical sequencing, and blunted or inappropriate affect (Schmahmann and Sherman 1998).

\section{Current Knowledge}

\section{Causes and Correlates of CAS}

The co-occurrence of these cognitive and affective symptoms arises from the disruption of neuroanatomical circuits connecting the cerebellum with frontal, parietal, temporal, and limbic cortices. Damage to these connections can occur in association with cerebellar infarct (Schmahmann and Sherman 1998), cerebellar atrophy associated with severe alcoholism (Fitzpatrick et al. 2008), cerebellar tumor or tumor resection (Levihson et al. 2000; Konczak 2005), trauma, neurodegenerative disorders, or cerebellitis. Affective symptoms have been associated with damage to the cerebellar vermis (Levihson et al. 1997). Lesions of the anterior lobe of the cerebellum tend to produce only minor changes in executive and visual-spatial functions. Children with a cognitive affective syndrome can also have autistic characteristics, and diagnosis of autism can be confounded by cerebellar lesions.

\section{References and Readings}

Schmahmann, J., \& Sherman, J. (1998). The cerebellar cognitive affective syndrome. Brain, 121, 561-579.

Schmahmann, J., Weilburg, J. D., Sherman, J. B., \& Janet, C. (2007). The neuropsychiatry of the cerebellum Insights from the clinic. Cerebellum, 6(3), 254-267. 